\title{
Nanoparticles-Emerging Potential for Managing Leukemia and Lymphoma
}

\author{
Raquel Vinhas, Rita Mendes, Alexandra R. Fernandes and Pedro V. Baptista* \\ UCIBIO, Departamento de Ciências da Vida, Faculdade de Ciências e Tecnologia, Universidade NOVA de Lisboa, \\ Caparica, Portugal
}

Nanotechnology has become a powerful approach to improve the way we diagnose and treat cancer. In particular, nanoparticles (NPs) possess unique features for enhanced sensitivity and selectivity for earlier detection of circulating cancer biomarkers. In vivo, NPs enhance the therapeutic efficacy of anticancer agents when compared with conventional chemotherapy, improving vectorization and delivery, and helping to overcome drug resistance. Nanomedicine has been mostly focused on solid cancers due to take

OPEN ACCESS

Edited by: Gianni Ciofani,

Politecnico di Torino, Italy

Reviewed by:

Attilio Marino,

Fondazione Istituto Italiano di

Tecnologia, Italy

Giada Graziana Genchi, Fondazione Istituto Italiano di

Tecnologia, Italy

Clara Mattu,

Politecnico di Torino, Italy

*Correspondence:

Pedro V. Baptista

pmvb@fct.unl.pt

Specialty section:

This article was submitted

to Nanobiotechnology,

a section of the journal

Frontiers in Bioengineering

and Biotechnology

Received: 22 October 2017 Accepted: 05 December 2017 Published: 18 December 2017

Citation:

Vinhas $R$, Mendes R, Fernandes $A R$ and Baptista PV (2017)

Nanoparticles - Emerging

Potential for Managing

Leukemia and Lymphoma.

Front. Bioeng. Biotechnol. 5:79. doi: 10.3389/fbioe.2017.00079 advantage from the enhanced permeability and retention (EPR) effect experienced by tissues in the close vicinity of tumors, which enhance nanomedicine's accumulation and, consequently, improve efficacy. Nanomedicines for leukemia and lymphoma, where EPR effect is not a factor, are addressed differently from solid tumors. Nevertheless, NPs have provided innovative approaches to simple and non-invasive methodologies for diagnosis and treatment in liquid tumors. In this review, we consider the state of the art on different types of nanoconstructs for the management of liquid tumors, from preclinical studies to clinical trials. We also discuss the advantages of nanoplatforms for theranostics and the central role played by NPs in this combined strategy.

Keywords: nanoparticles, leukemia, lymphoma, diagnostics, therapeutics, nanotheranostics, nanomedicine

\section{INTRODUCTION}

Traditional approaches for cancer management rely on centralized diagnostic platforms that can be complex, time consuming and set for the wide spectrum of patients and malignancies (Friedman et al., 2015). These traditional approaches have been developed toward big data generation from pools of patients and for the identification of suitable biomarkers and profiles, which are the basis for standardization of chemotherapy protocols (Zaimy et al., 2017). Such strategies allowed for the development of precision oncology, in which profile data of the cancer cells enable a tailored treatment of individual patients, and have become a crucial trend for cancer management.

Nanoscale particles have been playing a central role in the detection of cancer biomarkers, which is essential for the personalized assessment at the basis of precision treatment. Versatile structural and functional properties of the nanoparticles (NPs) offer the possibility for rapid, specific, and sensitive diagnostics, toward decentralized assessment and/or ambulatory follow-up. In what therapeutics are concerned, the size range of these nanoconstructs allows them to cross biological barriers more effectively that may be further improved by functionalizing the nanoconstructs' surface with specific ligands for precise delivery to the focus of disease (Lee et al., 2017). Moreover, NPs can work as therapeutic/imaging agents on their own or as carriers of multiple molecules that serve a specific function: tumor targeting, cancer cell ablation (via drug delivery or gene silencing), and real-time monitoring of cancer cells expansion or decay (Vinhas et al., 2015). This flexibility 
is crucial for theranostics, i.e., the simultaneous detection and therapy (Pedrosa et al., 2015). Most NP-based strategies have been directed at solid tumors, whereas the so-called non-solid tumors, such as lymphoma and leukemia, have not mobilized that much attention.

Conventional chemotherapy in hematological cancers is challenged by the poor selectivity, resulting in low therapeutic efficacy and pronounced adverse side effects. These issues may be overturned by innovative nanomedicine approaches. In this review, we shall focus on nanotechnology advances that support leukemia and lymphoma biomarker detection and targeted treatment. Hematological disorders are very good candidates for such targeting since the molecular basis of each subgroup of patients is very well defined by common chromosomal translocations, shared mutations in oncogenes, gene expression profiles, and immune phenotype. Moreover, a simple blood sample (liquid biopsy) provides access to the patient's full tumor profile, giving insightful information to support more focused therapeutic regimens-Figure 1. Unlike solid tumors that require NPs to reach the site of action, liquid tumors are spread throughout the

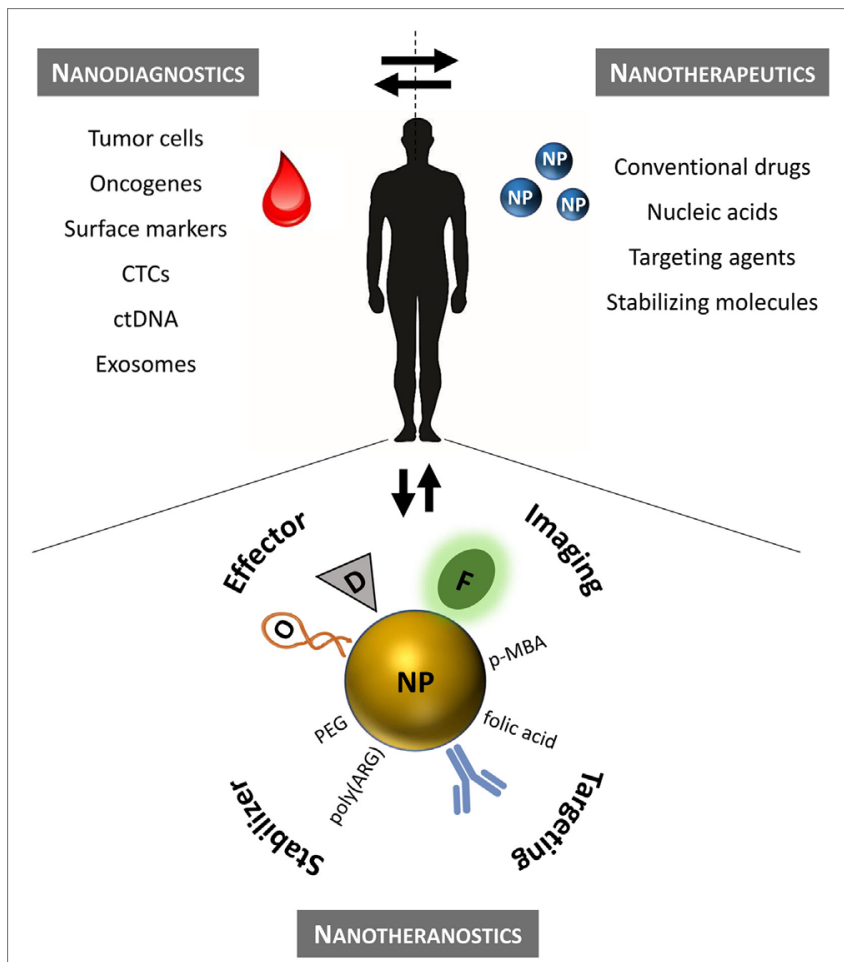

FIGURE 1 | Precision nanomedicine for the management of hematological disorders. Nanodiagnostics based on liquid biopsies to assess multiple leukemia/lymphoma biomarkers using NP (ex vivo analysis). Nanotherapeutics according to the patient's molecular profile (in vivo). Nanotheranostics combines diagnostics and therapeutics onto a single nanomaterial. Therapeutic efficacy may be improved for standard chemotherapeutics by efficient delivery to the target that can be monitored in real time. CTCs, circulating tumor cells; ctDNA, circulating tumor DNA; D, conventional drug; F, fluorophore; NP, nanoparticle; O, oligonucleotide; PEG, polyethylene glycol; p-MBA, p-mercaptobenzoic acid (Raman reporter); poly(ARG), poly-arginine peptide sequence. bloodstream. Most of the barriers that NPs face to reach solid tumors are not critical in liquid tumors since circulating tumor cells are freely exposed to these agents. However, while in circulation, NPs may still be opsonized by blood proteins followed by recognition by the mononuclear phagocyte system (Sriraman et al., 2014). As such, liquid tumors require slightly different diagnostic treatment and targeting strategies that shall be herein discussed.

\section{BACKGROUND OF LIQUID TUMORS}

Hematologic malignancies are the most common type of cancer among children and young adults, comprising leukemia, lymphoma, and myeloma, which affect the bone marrow, lymphatic system, and blood cells. The sequential stages of hematopoietic differentiation provide multiple opportunities for mutations to occur together with other disruptive events that lead to distinct tumor subtypes and clinical presentations ( $\mathrm{Hu}$ and Shilatifard, 2016). The heterogeneity of tumors of the hematopoietic and lymphoid tissues presents unique challenges for current diagnosis and treatment (Den Boer et al., 2009; Tiacci et al., 2015; Cazzola, 2016; Freeman and Gribben, 2016).

Leukemia is a clonal disorder originated in the bone marrow during hematopoiesis and is characterized by the unregulated proliferation of poorly differentiated white blood cells. Classification of the disease is based on the type of cell affected (myeloid or lymphoid) and the degree of cell proliferation (acute or chronic) (Hu and Shilatifard, 2016). Acute myeloid leukemia (AML) is the most common type in adults (with an annual incidence of 4.2 cases per 100,000 persons and an annual mortality rate of 2.8 per 100,000) and acute lymphocytic leukemia (ALL) that is more prevalent among pediatric patients (with an annual incidence of 1.7 cases per 100,000 persons and an annual mortality rate of 0.4 per 100,000) (Siegel et al., 2016). Chronic myeloid leukemia (CML) is a myeloproliferative disorder with an annual incidence of 1.8 cases per 100,000 adults, accounting for $15-20 \%$ of newly diagnosed cases of leukemia in adults, and an annual mortality rate of 0.4 cases per 100,000 adults (Jabbour and Kantarjian, 2012; Siegel et al., 2016).

Lymphoma originates in lymph nodes where the lymphoid lineage of hematopoiesis differentiates into B-cells, T-cells, or natural killer cells. Abnormal events include extensive cell proliferation, somatic mutations, and antibody class switching, that ultimately impair the immune system as a whole and the adaptive immune response in particular (Young and Staudt, 2013). It is the most common hematologic cancer in western countries with an annual incidence of 19.5 cases per 100,000 persons. The number of deaths is circa 5.9 per 100,000 per year, with the majority being associated with aberrant B-cells, tackled using immunomodulating agents, monoclonal antibodies, or kinase inhibitors.

\section{Molecular Characterization}

The foundational diagnosis of blood cancers is clinical, morphological, and immunophenotypic (usually via flow cytometry or immunocytochemistry) (Keeney et al., 2017), which is then followed by molecular characterization. Here, genetics is critical for the classification of these diseases and commonly assists 
risk stratification, from poor to favorable prognosis (Tasian and Hunger, 2017; Taylor et al., 2017). Treatment depends on the type of leukemia/lymphoma, disease stage, prior history of treatment, age, overall condition, and genetic profile. Most patients are treated with chemotherapy, and some may also undergo radiotherapy, stem cell transplantation, or targeted therapy (Zimmermann et al., 2016; Cuellar et al., 2017; Donato et al., 2017). CML is a good example of how hematological diseases have greatly benefited from the advance of cytogenetic and molecular methodologies. It was the first cancer in which a unique causative chromosomal abnormality was identified, $\mathrm{t}(9 ; 22)(\mathrm{q} 34 ; \mathrm{q} 11)$-Philadelphia chromosome $(\mathrm{Ph})$ - and the associated $B C R-A B L 1$ gene, providing a specific target for disease treatment (Vinhas et al., 2017a,b). Tyrosine kinase inhibitors (TKIs) target the dysregulated kinase activity of the fusion protein encoded by BCR-ABL1 (Campiotti et al., 2017; Patel et al., 2017).

\section{Liquid Biopsies in Leukemia and Lymphoma}

The diagnosis and molecular management of cancer are mainly performed on biopsies, which allow histological and genetic characterization of the tumor-a valuable tool to characterize subtype and correlate to therapy protocol and, eventually, predict therapeutic response (Crowley et al., 2013; Brock et al., 2015). In solid tumors, biopsies correspond to a small portion of the neoplasm, not representing the pronounced heterogeneity. Usually performed at a specific time point, the dynamics of the tumor and variation of the mutation pattern are neither assessed nor represent the whole cancer. Taking multiple biopsies from patients is impractical, costly, whose invasiveness presents considerable risk and, above all, tremendous discomfort to the patient. In what myeloid or lymphoid neoplasms are concerned, conventional diagnostics consist of blood counts and flow cytometry markers evaluation directly on peripheral blood cells, which are also the tumor itself (Kumar et al., 2016).

From a peripheral blood sample, it is possible to obtain circulation tumor cells (CTCs), circulating cell free DNA, and other circulating biomarkers, such as microRNAs (miRs) and vesicles, which can provide valuable information before and during treatment in a non-invasive way (Brock et al., 2015; Buder et al., 2016; Krishnamurthy et al., 2017; Ranuncolo, 2017; Zhang et al., 2017). Liquid biopsies for solid or liquid tumors are associated with circulating material-mainly CTCs, nucleic acids, and exosomes. In liquid tumors, a direct sample of tumor cells may be retrieved from peripheral blood enabling the usual assessment of leukocytes, hemoglobin, lymphocytes, and platelet levels, together with prognostic markers, regarding disease outcome and clinical decision-making (Gomes et al., 2017).

\section{NPS APPLICATIONS IN LIQUID TUMORS}

Cancer nanomedicine has raised the stakes in the detection of cancer biomarkers. Therapeutics could also benefit from nanotechnology-based strategies, which tend to be more selective than traditional chemotherapy. Among the plethora of nanoscale systems for cancer diagnostics and therapeutics, this review will focus on NPs. NPs are synthesized from diverse materials that, when at nanoscale, feature unique electronic, optical, and catalytic properties (Hasan, 2015; Nikalje, 2015; Tatar et al., 2016). There is a wide variety of NPs: organic, inorganic, and hybrid NPs. Due to their small size (optimal for intracellular uptake) and their large surface ratio (allowing functionalization with different biomolecules), NPs have been extensively used for diagnostic and therapeutic purposes (Tinkle et al., 2014; Venkatraman, 2014; Mendes et al., 2016). These nanosystems not only provide new prospects to overcome limitations associated with traditional drugs but also enable the incorporation of diagnostic and therapeutic functions onto a single platform, conveying nanotheranostics strategies for personalized medicine.

Organic NPs have been the most explored nanocarriers in cancer, including (i) dendrimers, which are highly branched, allowing the delivery of cargo and produced by controlled polymerization with a maximum of $10 \mathrm{~nm}$ of size; (ii) lipid-based NPs, such as liposomes and micelles that may carry a hydrophobic cargo and typically have $50-100 \mathrm{~nm}$ of size; and (iii) polymeric NPs, produced in a size range from 10 to $400 \mathrm{~nm}$ from synthetic, natural, hydrolytically, or enzymatically degradable polymers onto which a cytotoxic drug can be covalently attached, dissolved, encapsulated, or entrapped (Dawidczyk et al., 2014; Bhatia, 2016; Mendes et al., 2016). Organic NPs usually show high biocompatibility, whereas inorganic NPs provide advantages in terms of function and properties. The latter include carbon-based NPs (e.g., carbon nanotubes, buckyballs, and graphene), which feature remarkable strength and unique electrical properties (conducting, semi conducting, or insulating); metal NPs (e.g., gold, silver, and iron oxide); and quantum dots (QDs).

Among metal NPs, gold and ferromagnetic NPs have been explored the most. Gold NPs (AuNPs) are easy to synthesize and functionalize and have been used for drug and gene delivery, thermal ablation, and radiotherapy enhancement. AuNPs are also used in diagnostic applications due to their high sensitivity. Ferromagnetic NPs interest relies on their unique magnetic properties together with the possibility for surface functionalization, to be used as contrast agents for magnetic resonance imaging (MRI), hyperthermia, and drug delivery. QDs have been mainly explored for optical imaging since they show narrow and tunable emission spectra, a broad excitation spectrum and do not photobleach. QDs have also been proposed as therapeutic tool in conceptual in vitro and in vivo application. However, since they are synthesized from heavy metal elements, toxicity concerns have limited effective translation to the clinics.

\section{Nanodiagnostics in Liquid Tumors}

Traditionally, leukemia and lymphoma cells are detected through morphological analysis, immunohistochemistry, antibody microarrays, flow cytometry using fluorescent markers, fluorescence in situ hybridization, PCR, and DNA sequencing. One of the major issues in lymphoid and myeloid neoplasms diagnostics is the limit of detection of immature white blood cells, since their numbers are very low at an early stage of the disease. Because these cancer types are extremely common and aggressive, effective treatment depends greatly on the accuracy and sensitivity of 
diagnosis. Signal amplification coupled with NPs may be a viable approach for earlier detection. For example, fluorescent QDs and nanometer semiconductors, with their superior fluorescent features, have been used for optimized detection schemes. In addition to fluorescent enhancement, metal NPs unique physicochemical properties, including localized surface plasmon resonance (LSPR), photoluminescence, or superparamagnetic properties, have also been explored (Sharma et al., 2015) - see Table 1.

Aptamer-based nanodiagnostics systems have been proposed for acute leukemia via an antileukemia-thiolated aptamer (sgc8c) that specifically recognizes protein tyrosine kinase 7 (PTK7), an overexpressed transmembrane receptor in human T-cell ALL cells. Aptamers feature high affinity and selectivity toward their targets and have been used to functionalize AuNPs. These aptamer-NP systems have enhanced detection sensitivity, with a detection limit of 10 cells $/ \mathrm{mL}$, capable of differentiating leukemic from normal cells (Khoshfetrat and Mehrgardi, 2017). Yu et al. (2016) used aptamer-functionalized QDs to specifically detect leukemia cells in buffer and serum, whose toxicity was also evaluated in animal models, suggesting that the complex might be used both in vitro and in vivo (Table 1).

Chronic myeloid leukemia is associated with a unique chromosomal abnormality-BCR-ABL1 gene-which is used for unambiguous molecular diagnostics. A review of the literature indicates that nanodiagnostics strategies for CML have relied solely on gold NPs (see Table 1). Based on the AuNPs' LSPR, colorimetric detection of this molecular aberration has been achieved directly on total RNA purified from blood (Vinhas et al., 2016, 2017a). Cordeiro et al. (2016) used the AuNPs' capability to modulate fluorescence emission of nearby fluorophores to selectively detect BCR-ABL1 isoforms. AuNPs functionalized with specific ssDNA oligonucleotides allowed the identification of $B C R-A B L 1$ positive samples, discriminating between the e14a2 and e13a2 fusion transcripts.

Most targets in leukemia and lymphoma diagnostics are antigens, such as CD20 that is overexpressed by malignant of B-cells (Tazi et al., 2011). CD20 antibody (rituximab) has been applied to the treatment of lymphoma but it can also be used for lymphoid neoplasms diagnosis. Sahoo et al. (2017) used avidin-modified magnetic NPs (MNPs) functionalized with biotinylated anti-CD20 antibody and a permanent magnet to specifically detect and isolate lymphoma cells from mixed samples. Capolla and colleagues also used an anti-CD20 antibody functionalized into polymeric-fluorophore Cy5.5-labeled NPs for in vivo imaging directed at the diagnosis of B-cell malignancies. These NPs might also be used as a personalized treatment strategy by loading with drugs, becoming an attractive theranostics platform (Table 1) (Capolla et al., 2015). Besides CD20 antigens, CD45 and CD19 are the two most common surface proteins expressed by B-cells and used in diagnostic immunophenotyping. MacLaughlin et al. (2013) reported the use of surface-enhanced Raman scattering (SERS) for detection of these three surface proteins in an ex vivo model of malignant B-cells. AuNPs were functionalized with one of the specific monoclonal antibodies (anti-CD20, anti-CD45, or anti-CD19)

TABLE 1 | NPs-based diagnostic strategies for leukemia and lymphoma diseases.

\begin{tabular}{|c|c|c|c|c|}
\hline Type of NP & Tumor & Target & Approach & Reference \\
\hline $\begin{array}{l}\text { Aptamer-conjugated } \\
\text { Au-coated magnetic NPs }\end{array}$ & AML & PTK7 & $\begin{array}{l}\text { Aptamer (sgc8) recognizes PTK7 on screen-printed } \\
\text { graphene-nitrogen nanosheet electrode (N-GN) in vitro } \\
\text { cells and human blood plasma }\end{array}$ & $\begin{array}{l}\text { Khoshfetrat and } \\
\text { Mehrgardi (2017) }\end{array}$ \\
\hline $\begin{array}{l}\text { Hollow core photonic crystal } \\
\text { silver NPs }\end{array}$ & AML & Leukemia cells & $\begin{array}{l}\text { Portable monitoring of leukemic cells in vitro, using NPs } \\
\text { for SERS }\end{array}$ & Khetani et al. (2015) \\
\hline QDs-bsb-apt & ALL & PTK7 & Aptamer (sgc8) for imaging tumor cells in vitro and in vivo. & Yu et al. (2016) \\
\hline $\begin{array}{l}\text { Aptamer-functionalized } \\
\text { fluorescent silica NPs }\end{array}$ & ALL & PTK7 & $\begin{array}{l}\text { Sgc8-FSNPs specifically recognize leukemic cells via } \\
\text { fluorescence in vitro and in vivo }\end{array}$ & Tan et al. (2016) \\
\hline ssDNA-AuNPs & $\mathrm{CML}$ & e14a2 fusion transcript & $\begin{array}{l}\text { Gold nanoprobes hybridize to } B C R-A B L 1 \text { sequence } \\
\text { present in clinical samples (ex vivo) in colorimetric output }\end{array}$ & Vinhas et al. (2016) \\
\hline Gold nanobeacons & $\mathrm{CML}$ & $\begin{array}{l}\text { e13a2 and e14a2 } \\
\text { fusion transcripts }\end{array}$ & $\begin{array}{l}\text { FRET-based spectral codification for discrimination of the } \\
\text { two most common } B C R-A B L \text { fusion transcripts in vitro } \\
\text { leukemic cells }\end{array}$ & Cordeiro et al. (2016) \\
\hline Anti-CD20-polymeric NPs & CLL & CD20 & $\begin{array}{l}\text { In vivo imaging Cy5.5-labeled-probe for CD20-expressing } \\
\text { tumors based on fluorescence intensity }\end{array}$ & Capolla et al. (2015) \\
\hline PEG-coated SERS AuNPs & $\begin{array}{l}\text { Malignant B-cells } \\
\text { (leukemia/lymphoma) }\end{array}$ & $\begin{array}{l}\text { CD } 45, \text { CD19, and } \\
\text { CD20 }\end{array}$ & $\begin{array}{l}\text { Triplex spectra for SERS flow cytometry detecting anti- } \\
\text { CD20 SERS probes in clinical samples (ex vivo) }\end{array}$ & MacLaughlin et al. (2013) \\
\hline $\begin{array}{l}\text { Anti-CD20 antibody- } \\
\text { conjugated MNPs }\end{array}$ & Lymphoma cells & CD20 & $\begin{array}{l}\text { CTCs detection and isolation in vitro via MNPs } \\
\text { functionalized with anti-CD20 antibodies }\end{array}$ & Sahoo et al. (2017) \\
\hline $\begin{array}{l}\text { QDs-labeled oligonucleotide } \\
\text { probes }\end{array}$ & $\begin{array}{l}\text { Acute leukemia and } \\
\text { follicular lymphoma }\end{array}$ & $\begin{array}{l}\text { Myeloperoxidase, bcl- } \\
\text { 2, survivin, and XIAP }\end{array}$ & $\begin{array}{l}\text { Combination of oligonucleotide probes with spectral } \\
\text { imaging for multiplex ISH in FFPE human tissue biopsies }\end{array}$ & Tholouli et al. (2006) \\
\hline
\end{tabular}

AML, acute myeloid leukemia; ALL, acute lymphocytic leukemia; AuNP, gold nanoparticle; CML, chronic myeloid leukemia; CLL, chronic lymphocytic leukemia; PTK7, protein tyrosine kinase 7; FRET, fluorescence energy transfer; MNP, magnetic nanoparticles; NP, nanoparticle; PEG, polyethylene glycol; QD, quantum dot; SERS, surface-enhanced Raman scattering; ISH, in situ hybridization; FFPE, formalin-fixed paraffin embedded tissues; XIAP, X-linked inhibitor of apoptosis protein; CTC, circulation tumor cell. 
for SERS identification of target cells via flow cytometry. This strategy represents a significant step in the development of SERS immunophenotyping, improving sensitivity and specificity in blood cancer diagnosis (Table 1).

\section{NP-Based Strategies for Treatment of Liquid Tumors}

Current treatment for leukemia and lymphoma involve chemotherapy and radiation, which often induce long-term side effects and multidrug resistance. In addition, other more invasive strategies that require a matching donor, such as stem cell transplant, have also been proposed. Nanotechnology provides the possibility to selectively deliver a high payload of anticancer agents to malignant cells without damaging healthy cells or systemic toxicity, allowing them to reach critical tissue compartments, such as the lymph nodes and the bone marrow, otherwise inaccessible to drugs-see Table 2.

\section{Quantum Dots}

Quantum dots as nanocarriers have also been used to enhance antitumoral drug efficacy. Recently, $4 \mathrm{~nm}$ diameter cadmiumtelluride QDs were conjugated with wogonin, a natural flavonoid with antiproliferative activity against several cancers, to induce apoptosis and differentiation of tumor cells (Xu et al., 2014; Yang et al., 2014; Hu et al., 2015). These nanocomposites overcame multidrug-resistant leukemia by facilitating interaction between wogonin and abnormal cells (Huang et al., 2016).

\section{Metal NPs}

Numerous studies examine the robustness of metal NPs to potentiate current cancer therapy. Above all, AuNPs and MNPs exhibit good biocompatibility, low toxicity, biodegradability, and high volume-to-surface ratio. MNPs can be directed toward the tumor site using a magnetic field and are very good contrast agents in MRI (Estelrich et al., 2015; Gobbo et al., 2015). MNPs ranging from 12 to $23 \mathrm{~nm}$ have been combined with wogonin or cytarabine (mainly used to treat AML) to selectively tackle multidrug-resistant leukemia cells in vitro, acute leukemia, and Burkitt's lymphoma (Peng et al., 2016; Shahabadi et al., 2016).

AuNPs are highly customizable to deliver a drug specifically to a particular cell or tissue cell. In 2016, Gossai et al. functionalized $15 \mathrm{~nm}$ AuNPs with dsDNA oligonucleotides with a sequence corresponding to $B I R C 5$, a gene is overexpressed in CML cell lines, which was further loaded with dasatinib, a potent TKI frequently used against CML. Inside the cells, the target gene mRNA binds to the antisense oligonucleotide and releases the drug-conjugated DNA oligonucleotide proportionally to level of BIRC5 mRNA in those cells (Gossai et al., 2014). Other studies examined the in vitro efficacy of drug-coated AuNPs on AML treatment improvement using TKIs and fludarabine (Simon et al., 2015; Petrushev et al., 2016; Song et al., 2016). One of these systems took additional advantage of folate grafted to the NPs' surface. Because folate receptors are highly overexpressed on the surface of tumor cells these nanoconjugates were able to actively target AML cells (Song et al., 2016).

AuNPs provide protection against degradation by RNases, thus increasing circulating times and subsequent increase of the payload of drug delivered to cells. A recent study conjugated $14 \mathrm{~nm}$ AuNPs with hairpin ssDNA oligonucleotides that recognize the fusion transcript $B C R-A B L 1$, the molecular hallmark in CML to tackle this cancer (Suka et al., 2016). This strategy resulted in $B C R-A B L 1$ silencing and significant CML cell death, with high selectivity since it had no effect on cells that do not express the BCR-ABL1 transcript. Recently, Vinhas et al. $(2017 \mathrm{a}, \mathrm{b})$ combined the silencing potential of oligonucleotide functionalized AuNPs with imatinib. Combination of these agents selectively decreased cell survival, inducing loss of viability of imatinib-resistant $\mathrm{K} 562$ cells, showing great promise to overcome TKI resistance in $\mathrm{Ph}+$ diseases.

\section{Lipid NPs (LNPs)}

Lipid NPs show plenty of applications for drug delivery since they improve the stability of pharmaceuticals, be it lipophilic or hydrophilic molecules (Miao et al., 2017). LNPs have been used as vehicles for antileukemia all-trans retinoic acid (Silva et al., 2016), small interfering RNA (siRNA) technology to silence $\mathrm{Mcl}-1$ expression in mantle cell lymphoma in vitro models (Knapp et al., 2016), and target miRs overexpression in leukemic stem cells believed to be the cause of AML chemoresistance and relapse (Dorrance et al., 2015). Huang and colleagues used LNPs to actively target the potent topoisomerase I poison SN-38. Healthy T-cells were engineered to retain homing receptors that mirror lymphoma cell biodistribution and to be resistant to $\mathrm{SN}-38$. These live vectors were then used to carry lipid nanocapsules (LNCs) conjugated to SN-38 (LNC@ $\mathrm{SN}-38 ; 136 \mathrm{~nm}$ diameter) to reduce tumor burden and improve survival in murine models after only 2 weeks of treatment. Conveyed by these nanocapsules, SN-38 levels in lymph nodes were almost $1,000 \times$ higher than for the free SN-38 (Huang et al., 2015).

\section{Polymeric NPs}

Polymeric NPs, typically formed through the assembly of copolymers, have been used to deliver a spectrum of chemotherapeutic, diagnostic, and imaging agents in cancer (Kallinteri and Garnett, 2007). In one such examples, parthenolide (PTL), a preclinical agent that can eliminate resistant AML stem cells while preserving normal hematopoietic function, was first incorporated into mPEG-polylactic acid micelles, encapsulated in a protective degradable porous silicon (pSi), and coated with E-selectin thioaptamer (ESTA) to direct the particles toward the bone marrow (Zong et al., 2016). ESTA binds to the adhesion molecule E-selectin expressed exclusively by bone marrow endothelial cells (Winkler et al., 2012). The developed multistage vector successfully delivered PTL to the bone marrow of patient-derived AML xenografts. Two doses of the nanoconjugate, administered with a 2-week interval, were enough to impair leukemic stem cell function and AML burden in vivo (Zong et al., 2016).

Co-polymeric NPs were also used to deliver anticancer drug doxorubicin (DOX) against ALL. These DOX-coated NPs were further functionalized with CD19 antibodies to enhance their internalization via receptor-mediated endocytosis in CD19 positive ALL cells with minimal cytotoxicity toward healthy cells. Indeed, the $83 \mathrm{~nm}$ nanoconjugates were tested in ALL 
TABLE 2 | Recent studies (2015-2017) using nanoconjugates to improve leukemia and lymphoma management.

\begin{tabular}{|c|c|c|c|c|c|}
\hline Nanoconjugate & Effector molecule & Targeting agent & Imaging agent & Condition (in vitro/in vivo) & Reference \\
\hline QD-CdTe@Wogonin & Wogonin & n.a. & n.a. & $\begin{array}{l}\text { In vitro/in vivo multidrug-resistant leukemia } \\
\text { (K562-A02 cell line; leukemia-bearing mice) }\end{array}$ & Huang et al. (2016) \\
\hline MNP-Fe $\mathrm{O}_{4} @$ Wogonin & Wogonin & n.a. & MNPs as contrast agents for MRI & $\begin{array}{l}\text { In vitro multidrug-resistant leukemia (K562-A02 } \\
\text { cell line) }\end{array}$ & Peng et al. (2016) \\
\hline MNP-Fe $\mathrm{O}_{4} @ \mathrm{SiO}_{2} @$ Cytarabine & Cytarabine & n.a. & MNPs as contrast agents for MRI & $\begin{array}{l}\text { In vitro acute leukemia (HL60 and KG1 cell lines) } \\
\text { Burkitt's Iymphoma (Raji cell line) }\end{array}$ & Shahabadi et al. (2016) \\
\hline $\begin{array}{l}\text { AuNP@BIRC5@Dasatinib or AuNP@BIRC5@ } \\
\text { Cy5 (controlled drug release via presence of } \\
\text { BIRC5 mRNA) }\end{array}$ & Dasatinib & $\begin{array}{l}\text { BIRC5 dsDNA } \\
\text { oligonucleotide }\end{array}$ & Cy5 & $\begin{array}{l}\text { In vitro/in vivo CML (K562 cell line; K562-derived } \\
\text { murine xenografts) }\end{array}$ & Gossai et al. (2014) \\
\hline AuNP@FLT3-inhibitor & $\begin{array}{l}\text { Lestaurtinibmidostaurin } \\
\text { sorafenib quizartinib (FLT3 } \\
\text { inhibitors) }\end{array}$ & n.a. & n.a. & In vitro AML (THP1 and OCl-AML3 cell lines) & $\begin{array}{l}\text { Simon et al. (2015) and } \\
\text { Petrushev et al. (2016) }\end{array}$ \\
\hline AuNP@Fludarabine@Folic acid & Fludarabine phosphate & Folic acid & n.a. & In vitro $\mathrm{AML}$ (KG1 cell line) & Song et al. (2016) \\
\hline AuNP@PEG@e14a2 & $\begin{array}{l}\text { BCR-ABL1 ssDNA } \\
\text { oligonucleotide (e14a2) }\end{array}$ & n.a. & n.a. & In vitro CML (K562 cell line) & Vinhas et al. (2017b) \\
\hline AgNP@p-MBA@Rituximab & Rituximab & $\begin{array}{l}\text { Rituximab (detection } \\
\text { of CD20) }\end{array}$ & $\begin{array}{l}\text { p-Mercaptobenzoic acid ( } \mathrm{p}-\mathrm{MBA}) \\
\text { (Raman reporter and linker molecule) }\end{array}$ & $\begin{array}{l}\text { In vitro Burkitt's lymphoma (Daudi and Raji cell } \\
\text { lines) }\end{array}$ & Yao et al. (2016) \\
\hline $\begin{array}{l}\text { SLN@ATRA (cholesteryl butyrate-solid LNPS, } \\
\text { SLN) }\end{array}$ & All-trans retinoic acid (ATRA) & n.a. & n.a. & $\begin{array}{l}\text { In vitro acute leukemia (HL60, Jurkat and THP1 } \\
\text { cell lines) }\end{array}$ & Silva et al. (2016) \\
\hline LNP@Mcl1 & Mcl1 siRNA & n.a. & n.a. & $\begin{array}{l}\text { In vitro mantle cell lymphoma [JeKo-1 (normal) } \\
\text { and MAVER-1 (aggressive) cell lines] }\end{array}$ & Knapp et al. (2016) \\
\hline $\begin{array}{l}\text { LNP@antagomiR126@Anti-CD45.2 } \\
\text { (lipopolyplex NPs) }\end{array}$ & AntagomiR-126 & CD45.2 antibody & n.a. & $\begin{array}{l}\text { In vitro/in vivo AML (human primary cells; murine } \\
\text { model) }\end{array}$ & Dorrance et al. (2015) \\
\hline $\begin{array}{l}\text { T-cells@LNC@SN-38@PEG [lipid } \\
\text { nanocapsule (LNC)] }\end{array}$ & $\begin{array}{l}\text { Topoisomerase I poison } \\
\text { SN-38 }\end{array}$ & $\begin{array}{l}\text { Healthy primary } \\
\text { T-cells (live vector) }\end{array}$ & n.a. & In vivo lymphoma (murine model) & Huang et al. (2015) \\
\hline $\begin{array}{l}\text { Nanopolymer-PTL@pSi@ESTA [micellar NPs } \\
\text { protected by porous silicon (pSi) coating] }\end{array}$ & Parthenolide (PTL) & $\begin{array}{l}\text { E-selectin } \\
\text { thioaptamer (ESTA) } \\
\text { (bone marrow- } \\
\text { directed aptamer) }\end{array}$ & n.a. & In vivo AML (patient-derived murine xenografts) & Zong et al. (2016) \\
\hline Nanopolymer@DOX@Anti-CD19 & DOX & CD19 antibody & n.a. & $\begin{array}{l}\text { In vitro/in vivo ALL (REH and RS4; } 11 \text { cell lines: } \\
\text { human-derived murine xenografts) }\end{array}$ & Krishnan et al. (2015) \\
\hline $\begin{array}{l}\text { MSN@DOX@PEG@Rituximab (pH-controlled } \\
\text { delivery via MSNs) }\end{array}$ & DOX and rituximab & $\begin{array}{l}\text { Rituximab (CD20 } \\
\text { antibody) }\end{array}$ & n.a. & $\begin{array}{l}\text { In vitro/in vivo B-cell lymphoma (Raji and Daudi } \\
\text { cell lines; murine model) }\end{array}$ & Zhou et al. (2017) \\
\hline $\begin{array}{l}\text { DNP@BCR-FITC@polyArg-BCL2 [diatomite } \\
\text { NPs (DNPs)] }\end{array}$ & BCL2 siRNA & $\begin{array}{l}\text { Idiotype-peptide } \\
\text { specific BCR }\end{array}$ & FITC & In vitro lymphoma (murine A20 cell line) & Martucci et al. (2016) \\
\hline $\begin{array}{l}\text { Rituximab-ABX-Alexa750 (rituximab binds to } \\
\text { albumin of ABX, NP-albumin-bound PTX) }\end{array}$ & PTX and rituximab & $\begin{array}{l}\text { Rituximab (CD20 } \\
\text { antibody) }\end{array}$ & AF750 & $\begin{array}{l}\text { In vitro/in vivo B-cell lymphoma (Daudi cells; } \\
\text { human B-cell lymphoma murine model) }\end{array}$ & Nevala et al. (2017) \\
\hline
\end{tabular}

AF750, alexafluor 750; AML, acute myeloid leukemia; CML, chronic myeloid leukemia; DOX, doxorubicin; n.a., not applicable; PEG, polyethylene glycol; polyArg, peptide sequence with nine arginines; PTX, paclitaxel; NP, nanoparticle;

ALL, acute lymphocytic leukemia; QD, quantum dot; MNP, magnetic NP; BCR, B-cell receptor; LNP, lipid NP; siRNA, small interfering RNA; MSN, mesoporous silica NP; ABX, ambraxane. 
mice, exhibiting higher therapeutic efficacy and reduced systemic toxicity than that of free DOX (Krishnan et al., 2015).

\section{Mesoporous Silica NPs (MSNs) toxic side effects. \\ FUTURE PERSPECTIVE ON NANOTECHNOLOGY FOR HEMATOLOGICAL DISEASES}

Mesoporous silica NPs are also suitable for nanotherapeutics in cancer due to their unique porous structure, tunable pore size, and the ability to release drugs in response to $\mathrm{pH}$, temperature, light, redox reactions, enzymes, or biomolecules (Cheng et al., 2017; Lin et al., 2017; Yan et al., 2017; Zhan et al., 2017). Zhou et al. (2017) incorporated DOX into the pores of $\mathrm{pH}$-sensitive MSNs, whose $\mathrm{pH}$-responsive mechanism enabled the controlled release of low levels of drug at a physiological $\mathrm{pH}$ and efficient intracellular release under more acidic conditions, characteristic of endosomal and lysosomal environments. To improve targeting of MSN@DOX to tumor cells, these NPs were functionalized with rituximab via an avidin-biotin system. In vitro tests showed that the targeted system was internalized more efficiently by $\mathrm{CD} 20$ positive than by $\mathrm{CD} 20$ negative cells. In vivo tests confirmed the effective deliver of DOX to lymphoma B-cells, inducing cell apoptosis and inhibiting tumor growth with minimal

\section{Nanotheranostics}

Many features of NPs herein described are particularly attractive for biomarker detection and simultaneous abnormal cells ablation, making them suitable candidates for nanotheranostics (Chen et al., 2017). In liquid cancers, tumor cells are free in circulation, requiring specific active targeting. However, confined tumor sites are also present, such as the bone marrow and/or lymphoid tissues, nanosystems profiting from the EPR effect may be of extreme relevance in tackling these niches. This way, therapeutic nanoconjugates may accumulate at tumor locations, where subsequent active targeting to cancer cells may be achieved (Lammers et al., 2012). A few preclinical studies on lymphoma nanotheranostics using metal NPs, diatomite NPs (DNPs), and nanoantibodies (nanobodies) have already been reported-see Table 2.

For example, $50 \mathrm{~nm}$ AgNPs have been conjugated to pmercaptobenzoic acid-a Raman reporter and linker molecule, together with rituximab, for B-cell lymphoma targeting and ablation - to allow non-invasive detection of living cells without labeling based on SERS. In addition, these silver nanostructures were tuned to enhance SERS signals from single molecule, thus allowing multiplexing. The so designed Ag nanoconstruct was capable to simultaneously detect CD20 positive single lymphoma cell and eliminate them with high selectivity (Yao et al., 2016).

Diatomite NPs, silica-based NPs of irregular shape and mean size of approximately $200 \mathrm{~nm}$, were also applied in the management of B-cell lymphoma (Martucci et al., 2016). These DNPs have been modified to actively target the hypervariable region of surface immunoglobulin B-cell receptor (BCR) toward fluorescence-based monitorization via FITC and confocal microscopy/ flow cytometry. These conjugates also simultaneously recognize and downregulate the antiapoptotic factor B-cell lymphoma/ leukemia 2 (Bcl2) mediated by siRNA therein encapsulated. The nanostructure successfully mediated in vitro BCL2 gene silencing in a target selective manner. This approach could be applied in the follow-up of lymphoma/leukemia patients.

Another example of nanotheranostics using a nano-antibody composed of rituximab conjugated to an NP albumin-bound paclitaxel [ambraxane (ABX)] has been developed toward decimate B-cell lymphoma (Nevala et al., 2017). In vivo imaging of the tumor burden reduction was achieved by labeling the ABX with alexafluor 750 . The $160 \mathrm{~nm}$ nanocontruct retained the cytotoxicity of $\mathrm{ABX}$ and the $\mathrm{CD} 20$ affinity of rituximab, both in vitro and in vivo. Moreover, by combining both antibodies at nanoscale, higher therapeutic efficacy was achieved when compared with $\mathrm{ABX}$ or rituximab alone.

\section{Current Limitations of NPs and Leukemia/ Lymphoma In Vivo Models}

Thus far, nanoformulations have enhanced biomarker detection, providing simpler assays with higher sensitivity. Nanomedicines have also been shown to improve the efficacy-toxicity ratio of anticancer agents, thus offering the possibility to monitor diagnostics and treatment of liquid tumors in real time. However, the success of nanomedicines at a preclinical stage depends greatly on the availability of in vivo tumor models that mimic the real human tumor environment. Leukemia/lymphoma models show several obstacles since the pathogenesis of the disease in murine models is not relevant to most human cases; also, these models fail to replicate the complex microenvironment from which these human cancers arise, and do not embody their genetic and molecular heterogeneity (Cook and Pardee, 2013; Kohnken et al., 2017). Xenografts mitigate some of these issues, but they are usually conducted on immunocompromised mice to avoid immune rejection of human cells, which excludes the effects of the immune system on tumor expansion and on NPs efficacy and targeting.

The variability of experimental conditions between different preclinical studies using NPs to tackle leukemia and lymphoma also contribute to their reduced clinical impact. There is a lack of standardized manufacturing procedures and controls, recognized by regulatory agencies-US Food and Drug Administration (FDA) or the European Medicines Agency-for clinical translation of nanoscale diagnostic assays and treatment. In addition, there have been a deficiency on in vivo toxicity, stability, and biodistribution studies that are essential to determine NPs ability as delivery vehicle, imaging, or therapeutic agent (Dawidczyk et al., 2014; Shi et al., 2016).

\section{Clinical Trials}

Preclinical studies of nanomedicines against hematological malignancies hold great promise but thus far only but a few have reached the clinical trial stage. Of these, liposomal nanoformulations show some impact in blood malignancies, and three liposomal formulations for the treatment of leukemia have reached advanced clinical trials. In 2012, liposomal vincristine 
sulfate (Marqibo ${ }^{\circledR}$ ) was the first nanoformulation to get approval by the FDA to treat Ph+ ALL in adults that relapse or that do not respond to at least two antileukemia drugs. Vincristine inhibits microtubule formation in mitotic spindle, resulting in an arrest of dividing tumor cells at the metaphase stage and is a standard component of chemotherapy regimens used to treat ALL and other lymphoid malignancies for over 50 years. However, vincristine's free version induces major side effects. Encapsulation in a sphingomyelin/cholesterol-based liposome changed vincristine pharmacokinetics dramatically, leading to a slow release from vector and delivery to tissues more efficiently, allowing administration of higher doses (ClinicalTrials.gov Identifier: NCT01439347) (Douer, 2016). Marqibo ${ }^{\circledR}$ has also completed phase I clinical trials for the treatment of pediatric ALL (ClinicalTrials.gov Identifier NCT01222780) (Shah et al., 2016).

Other examples of promising nanomedicines currently in advanced stage of development include the following: (i) CPX351, a liposomal formulation of cytarabine and daunorubicin, that yielded interesting results in phase III clinical trials for the treatment of high-risk AML. In fact, CPX-351 significantly improved overall survival, event-free survival, and response rates in comparison with the standard regimen of cytarabine and daunorubicin (ClinicalTrials.gov Identifier: NCT02286726); (ii) annamycin, an anthracycline intended for the treatment of relapsed or refractory leukemia, has also been encapsulated in a liposome and submitted to phase I/II multicenter clinical trials. The drug was well tolerated and showed encouraging antileukemic activities against ALL (ClinicalTrials.gov Identifier: NCT00271063) (Wetzler et al., 2013).

Future studies have been proposed focusing on the combination of these nanoformulations with other antileukemic drugs,

\section{REFERENCES}

Bhatia, S. (ed.). (2016). "Nanoparticles types, classification, characterization, fabrication methods and drug delivery applications," in Natural Polymer Drug Delivery Systems: Nanoparticles, Plants, and Algae (Switzerland: Springer International Publishing), 33-93.

Brock, G., Castellanos-Rizaldos, E., Hu, L., Coticchia, C., and Skog, J. (2015). Liquid biopsy for cancer screening, patient stratification and monitoring. Transl. Cancer Res. 4, 280-290. doi:10.3978/j.issn.2218-676X.2015.06.05

Buder, A., Tomuta, C., and Filipits, M. (2016). The potential of liquid biopsies. Curr. Opin. Oncol. 28, 130-134. doi:10.1097/CCO.0000000000000267

Campiotti, L., Suter, M. B., Guasti, L., Piazza, R., Gambacorti-Passerini, C., Grandi, A. M., et al. (2017). Imatinib discontinuation in chronic myeloid leukaemia patients with undetectable BCR-ABL transcript level: a systematic review and a meta-analysis. Eur. J. Cancer 77, 48-56. doi:10.1016/j.ejca.2017.02.028

Capolla, S., Garrovo, C., Zorzet, S., Lorenzon, A., Rampazzo, E., Spretz, R., et al. (2015). Targeted tumor imaging of anti-CD20-polymeric nanoparticles developed for the diagnosis of B-cell malignancies. Int. J. Nanomedicine 10, 4099-4109. doi:10.2147/IJN.S78995

Cazzola, M. (2016). Introduction to a review series: the 2016 revision of the WHO classification of tumors of hematopoietic and lymphoid tissues. Blood 127, 2361-2364. doi:10.1182/blood-2016-03-657379

Chen, H., Zhang, W., Zhu, G., Xie, J., and Chen, X. (2017). Rethinking cancer nanotheranostics. Nat. Rev. Mater. 2, 17024. doi:10.1038/natrevmats.2017.24

Cheng, W., Nie, J., Xu, L., Liang, C., Peng, Y., Liu, G., et al. (2017). pH-Sensitive delivery vehicle based on folic acid-conjugated polydopamine-modified mesoporous silica nanoparticles for targeted cancer therapy. ACS Appl. Mater. Interfaces 9, 18462-18473. doi:10.1021/acsami.7b02457 namely, TKIs, and incorporate new classes of therapeutic agents, such as, siRNA, miRs, ssDNA, and gene editing.

\section{CONCLUSION}

Cancer nanomedicine has been evolving steadily for the past 10-15 years, putting forward solutions for more accurate diagnostics, including molecular characterization in rapid point-of-need platforms, and radical new approaches of smart NPs for therapy. However, these advances have yet to prove efficacy in the clinics, before eventually becoming routine substitutes of traditional chemo/radio therapy. The vast majority of these nanomedicines have focused on tackling solid tumors, with the characteristic implantation, invasion, inflammatory response, and metastasis. Liquid tumors have been somehow neglected, but new strategies for both diagnostics and therapeutics are slowly gaining pace. Nanomedicine holds great promise to tackle liquid tumors, both at the local environment (e.g., bone marrow) and in circulation since most malignant cells are spread throughout the body via the blood and lymph. Newer and smarter NP-based approaches are required to eliminate these cancer cells with increased efficacy and specificity.

\section{AUTHOR CONTRIBUTIONS}

$\mathrm{PB}$ and $\mathrm{AF}$ outlined the project. All the authors reviewed the literature, drafted the manuscript, and approved it for publication.

\section{FUNDING}

This work was partially supported by FCT/MEC to UCIBIO (UID/Multi/04378/2013); RV by PD/BD/52211/2013; RM by PTDC/QEQ-MED/1902/2014.

Cook, G. J., and Pardee, T. S. (2013). Animal models of leukemia: any closer to the real thing? Cancer Metastasis Rev. 32, 63-76. doi:10.1007/s10555-012-9405-5

Cordeiro, M., Giestas, L., Lima, J. C., and Baptista, P. M. V. (2016). BioCode gold-nanobeacon for the detection of fusion transcripts causing chronic myeloid leukemia. J. Nanobiotechnology 14, 38. doi:10.1186/s12951-016-0192-y

Crowley, E., Di Nicolantonio, F., Loupakis, F., and Bardelli, A. (2013). Liquid biopsy: monitoring cancer-genetics in the blood. Nat. Rev. Clin. Oncol. 10, 472-484. doi:10.1038/nrclinonc.2013.110

Cuellar, S., Vozniak, M., Rhodes, J., Forcello, N., and Olszta, D. (2017). BCR-ABL1 tyrosine kinase inhibitors for the treatment of chronic myeloid leukemia. J. Oncol. Pharm. Pract. doi:10.1177/1078155217710553

Dawidczyk, C. M., Russell, L. M., and Searson, P. C. (2014). Nanomedicines for cancer therapy: state-of-the-art and limitations to pre-clinical studies that hinder future developments. Front. Chem. 2:69. doi:10.3389/fchem.2014.00069

Den Boer, M. L., van Slegtenhorst, M., De Menezes, R. X., Cheok, M. H., BuijsGladdines, J. G., Peters, S. T., et al. (2009). A subtype of childhood acute lymphoblastic leukaemia with poor treatment outcome: a genome-wide classification study. Lancet Oncol. 10, 125-134. doi:10.1016/S1470-2045(08)70339-5

Donato, E. M., Fernández-Zarzoso, M., and De La Rubia, J. (2017). Immunotherapy for the treatment of Hodgkin lymphoma. Expert Rev. Hematol. 10, 417-423. doi:10.1080/17474086.2017.1313701

Dorrance, A. M., Neviani, P., Ferenchak, G. J., Huang, X., Nicolet, D., Maharry, K. S., et al. (2015). Targeting leukemia stem cells in vivo with antagomiR-126 nanoparticles in acute myeloid leukemia. Leukemia 29, 2143-2153. doi:10.1038/leu. 2015.139

Douer, D. (2016). Efficacy and safety of vincristine sulfate liposome injection in the treatment of adult acute lymphocytic leukemia. Oncologist 21, 840-847. doi:10.1634/theoncologist.2015-0391 
Estelrich, J., Sánchez-Martín, M. J., and Busquets, M. A. (2015). Nanoparticles in magnetic resonance imaging: from simple to dual contrast agents. Int. J. Nanomedicine 10, 1727-1741. doi:10.2147/IJN.S76501

Freeman, C. L., and Gribben, J. G. (2016). Immunotherapy in chronic lymphocytic leukaemia (CLL). Curr. Hematol. Malig. Rep. 11, 29-36. doi:10.1007/ s11899-015-0295-9

Friedman, A. A., Letai, A., Fisher, D. E., and Flaherty, K. T. (2015). Precision medicine for cancer with next-generation functional diagnostics. Nat. Rev. Cancer 15, 747-756. doi: $10.1038 /$ nrc4015

Gobbo, O. L., Sjaastad, K., Radomski, M. W., Volkov, Y., and Prina-Mello, A. (2015). Magnetic nanoparticles in cancer theranostics. Theranostics 5, 1249-1263. doi:10.7150/thno.11544

Gomes, L. C., Evangelista, F. C. G., Sousa, L. P., Araujo, S., Carvalho, M. D. G., and Sabino, A. D. P. (2017). Prognosis biomarkers evaluation in chronic lymphocytic leukemia. Hematol. Oncol. Stem Cell Ther. 10, 57-62. doi:10.1016/j. hemonc.2016.12.004

Gossai, N. P., Naumann, J. A., Li, N.-S., Zamora, E. A., Gordon, D. J., Piccirilli, J. A., et al. (2014). Drug conjugated nanoparticles activated by cancer cell specific mRNA. Oncotarget 7, 38243-38256. doi:10.18632/oncotarget.9430

Hasan, S. (2015). A review on nanoparticles: their synthesis and types. Res. J. Recent Sci. 4, 1-3.

Hu, C., Xu, M., Qin, R., Chen, W., and Xu, X. (2015). Wogonin induces apoptosis and endoplasmic reticulum stress in HL-60 leukemia cells through inhibition of the PI3K-AKT signaling pathway. Oncol. Rep. 33, 3146-3154. doi:10.3892/ or.2015.3896

Hu, D., and Shilatifard, A. (2016). Epigenetics of hematopoiesis and hematological malignancies. Genes Dev. 30, 2021-2041. doi:10.1101/gad.284109.116

Huang, B., Abraham, W. D., Zheng, Y., Bustamante Lopez, S. C., Luo, S. S., and Irvine, D. J. (2015). Active targeting of chemotherapy to disseminated tumors using nanoparticle-carrying T cells. Sci. Transl. Med. 7, 291ra94. doi:10.1126/ scitranslmed.aaa5447

Huang, B., Liu, H., Huang, D., Mao, X., Hu, X., Jiang, C., et al. (2016). Apoptosis induction and imaging of cadmium-telluride quantum dots with wogonin in multidrug-resistant leukemia K562/A02 cell. J. Nanosci. Nanotechnol. 16, 2499-2503. doi:10.1166/jnn.2016.10792

Jabbour, E., and Kantarjian, H. (2012). Chronic myeloid leukemia: 2012 update on diagnosis, monitoring, and management. Am. J. Hematol. 87, 1037-1045. doi:10.1002/ajh.23282

Kallinteri, P., and Garnett, M. C. (2007). "Polymeric nanoparticles for drug delivery," in Nanotechnologies for the Life Sciences (Germany: Wiley-VCH Verlag GmbH \& Co. KGaA), 409-470. doi:10.1002/9783527610419.ntls0115

Keeney, M., Hedley, B. D., and Chin-Yee, I. H. (2017). Flow cytometryrecognizing unusual populations in leukemia and lymphoma diagnosis. Int. J. Lab. Hematol. 39, 86-92. doi:10.1111/ijlh.12666

Khetani, A., Momenpour, A., Alarcon, E. I., and Anis, H. (2015). Hollow core photonic crystal fiber for monitoring leukemia cells using surface enhanced Raman scattering (SERS). Biomed. Opt. Express 23, 8992-8998. doi:10.1364/ OE.23.04599

Khoshfetrat, S. M., and Mehrgardi, M. A. (2017). Amplified detection of leukemia cancer cells using an aptamer-conjugated gold-coated magnetic nanoparticles on a nitrogen-doped graphene modified electrode. Bioelectrochemistry 114, 24-32. doi:10.1016/j.bioelechem.2016.12.001

Knapp, C. M., He, J., Lister, J., and Whitehead, K. A. (2016). Lipidoid nanoparticle mediated silencing of Mcl-1 induces apoptosis in mantle cell lymphoma. Exp. Biol. Med. 241, 1-7. doi:10.1177/1535370216640944

Kohnken, R., Porcu, P., and Mishra, A. (2017). Overview of the use of murine models in leukemia and lymphoma research. Front. Oncol. 7:22. doi:10.3389/ fonc.2017.00022

Krishnamurthy, N., Spencer, E., Torkamani, A., and Nicholson, L. (2017). Liquid biopsies for cancer: coming to a patient near you. J. Clin. Med. 6, 3. doi:10.3390/ jcm6010003

Krishnan, V., Xu, X., Kelly, D., Snook, A., Waldman, S. A., Mason, R. W., et al. (2015). CD19-targeted nanodelivery of doxorubicin enhances therapeutic efficacy in B-cell acute lymphoblastic leukemia. Mol. Pharm. 12, 2101-2111. doi:10.1021/acs.molpharmaceut.5b00071

Kumar, B., Garcia, M., Murakami, J. L., and Chen, C. C. (2016). Exosome-mediated microenvironment dysregulation in leukemia. Biochim. Biophys. Acta 1863, 464-470. doi:10.1016/j.bbamcr.2015.09.017
Lammers, T., Kiessling, F., Hennink, W. E., and Storm, G. (2012). Drug targeting to tumors: principles, pitfalls and (pre-) clinical progress. J. Control Release 161, 175-187. doi:10.1016/j.jconrel.2011.09.063

Lee, J. J., Yazan, L. S., and Abdullah, C. A. C. (2017). A review on current nanomaterials and their drug conjugate for targeted breast cancer treatment. Int. J. Nanomedicine 12, 2373-2384. doi:10.2147/IJN.S127329

Lin, J. T., Liu, Z. K., Zhu, Q. L., Rong, X. H., Liang, C. L., Wang, J., et al. (2017). Redox-responsive nanocarriers for drug and gene co-delivery based on chitosan derivatives modified mesoporous silica nanoparticles. Colloids Surf. B Biointerfaces 155, 41-50. doi:10.1016/j.colsurfb.2017.04.002

MacLaughlin, C. M., Mullaithilaga, N., Yang, G., Ip, S. Y., Wang, C., and Walker, G. C. (2013). Surface-enhanced Raman scattering dye-labeled Au nanoparticles for triplexed detection of leukemia and lymphoma cells and SERS flow cytometry. Langmuir 29, 1908-1919. doi:10.1021/la303931c

Martucci, N. M., Migliaccio, N., Ruggiero, I., Albano, F., Calì, G., Romano, S., et al. (2016). Nanoparticle-based strategy for personalized B-cell lymphoma therapy. Int. J. Nanomedicine 11, 6089-6101. doi:10.2147/IJN.S118661

Mendes, R., Carreira, B., Baptista, P. V., and Fernandes, A. R. (2016). Non-small cell lung cancer biomarkers and targeted therapy - two faces of the same coin fostered by nanotechnology. Expert Rev. Precis. Med. Drug Dev. 1, 155-168. doi:10.1080/23808993.2016.1159914

Miao, L., Guo, S., Lin, C. M., Liu, Q., and Huang, L. (2017). Nanoformulations for combination or cascade anticancer therapy. Adv. Drug Deliv. Rev. 115, 3-22. doi:10.1016/j.addr.2017.06.003

Nevala, W. K., Butterfield, J. T., Sutor, S. L., Knauer, D. J., and Markovic, S. N. (2017). Antibody-targeted paclitaxel loaded nanoparticles for the treatment of CD20+ B-cell lymphoma. Sci. Rep. 7, 45682. doi:10.1038/srep45682

Nikalje, A. P. (2015). Nanotechnology and its applications in medicine. Med. Chem. 5, 81-89. doi:10.4172/2161-0444.1000247

Patel, A. B., Wilds, B. W., and Deininger, M. W. (2017). Treating the chronicphase chronic myeloid leukemia patient: which TKI, when to switch and when to stop? Expert Rev. Hematol. 10, 659-674. doi:10.1080/17474086.20 17.1330144

Pedrosa, P., Vinhas, R., Fernandes, A., and Baptista, P. (2015). Gold nanotheranostics: proof-of-concept or clinical tool? Nanomaterials 5, 1853-1879. doi:10.3390/nano5041853

Peng, M.-X., Wang, X.-Y., Wang, F., Wang, L., Xu, P.-P., and Chen, B. (2016). Apoptotic mechanism of human leukemia K562/A02 cells induced by magnetic ferroferric oxide nanoparticles loaded with wogonin. Chin. Med. J. (Engl.) 129, 2958. doi:10.4103/0366-6999.195466

Petrushev, B., Simon, T., Berce, C., Dima, D., Tanase, A., Zdrenghea, M., et al. (2016). Gold nanoparticles enhance the effect of tyrosine kinase inhibitors in acute myeloid leukemia therapy. Int. J. Nanomedicine 11, 641-660. doi:10.2147/ IJN.S94064

Ranuncolo, S. M. (2017). Liquid biopsy in liquid tumors. J. Cancer Ther. 8, 302-320. doi:10.4236/jct.2017.83026

Sahoo, S. L., Liu, C.-H., and Wu, W.-C. (2017). Lymphoma cell isolation using multifunctional magnetic nanoparticles: antibody conjugation and characterization. RSC Adv. 7, 22468-22478. doi:10.1039/C7RA02084H

Shah, N.N., Merchant, M. S., Cole, D. E., Jayaprakash, N., Bernstein, D., Delbrook, C., et al. (2016). Vincristine sulfate liposomes injection (VSLI, Marqibo ${ }^{\circledR}$ ): results from a phase I study in children, adolescents, and young adults with refractory solid tumors or leukemias. Pediatr. Blood Cancer 63, 997-1005. doi:10.1002/ pbc. 25937

Shahabadi, N., Falsafi, M., and Mansouri, K. (2016). Improving antiproliferative effect of the anticancer drug cytarabine on human promyelocytic leukemia cells by coating on Fe3O4@SiO2 nanoparticles. Colloids Surf. B Biointerfaces 141, 213-222. doi:10.1016/j.colsurfb.2016.01.054

Sharma, H., Mishra, P. K., Talegaonkar, S., and Vaidya, B. (2015). Metal nanoparticles: a theranostic nanotool against cancer. Drug Discov. Today 20, 1143-1151. doi:10.1016/j.drudis.2015.05.009

Shi, J., Kantoff, P. W., Wooster, R., and Farokhzad, O. C. (2016). Cancer nanomedicine: progress, challenges and opportunities. Nat. Rev. Cancer 17, 20-37. doi:10.1038/nrc.2016.108

Siegel, R. L., Miller, K. D., and Jemal, A. (2016). Cancer statistics. CA Cancer J. Clin. 66, 7-30. doi:10.3322/caac.21332

Silva, E. L., Lima, F. A., Carneiro, G., Ramos Jonas, P., Gomes, D. A., de SouzaFagundes, E. M., et al. (2016). Improved in vitro antileukemic activity of 
all-trans retinoic acid loaded in cholesteryl butyrate solid lipid nanoparticles. J. Nanosci. Nanotechnol. 16, 1291-1300. doi:10.1166/jnn.2016.11677

Simon, T., Tomuleasa, C., Bojan, A., Berindan-Neagoe, I., Boca, S., and Astilean, S. (2015). Design of FLT3 inhibitor - gold nanoparticle conjugates as potential therapeutic agents for the treatment of acute myeloid leukemia. Nanoscale Res. Lett. 10, 466. doi:10.1186/s11671-015-1154-2

Song, S., Hao, Y., Yang, X., Patra, P., and Chen, J. (2016). Using gold nanoparticles as delivery vehicles for targeted delivery of chemotherapy drug fludarabine phosphate to treat hematological cancers. J. Nanosci. Nanotechnol. 16, 2582-2586. doi:10.1166/jnn.2016.12349

Sriraman, S. K., Aryasomayajula, B., and Torchilin, V. P. (2014). Barriers to drug delivery in solid tumors. Tissue Barriers 2, e29528. doi:10.4161/tisb.29528

Suka, J. S., Xua, Q., Kima, N., Hanesa, J., and Ensign, L. M. (2016). PEGylation as a strategy for improving nanoparticle-based drug and gene delivery. Adv. Drug Deliv. Rev. 99, 28-51. doi:10.1016/j.addr.2015.09.012

Tan, J., Yang, N., Hu, Z., Su, J., Zhong, J., Yang, Y., et al. (2016). Aptamerfunctionalized fluorescent silica nanoparticles for highly sensitive detection of leukemia cells. Nanoscale Res. Lett. 11, 298. doi:10.1186/s11671016-1512-8

Tasian, S. K., and Hunger, S. P. (2017). Genomic characterization of paediatric acute lymphoblastic leukaemia: an opportunity for precision medicine therapeutics. Br. J. Haematol. 176, 867-882. doi:10.1111/bjh.14474

Tatar, A. S., Nagy-Simon, T., Tomuleasa, C., Boca, S., and Astilean, S. (2016). Nanomedicine approaches in acute lymphoblastic leukemia. J. Control Release 238, 123-138. doi:10.1016/j.jconrel.2016.07.035

Taylor, J., Xiao, W., and Abdel-Wahab, O. (2017). Diagnosis and classification of hematologic malignancies on the basis of genetics. Blood 130, 410-423. doi:10.1182/blood-2017-02-734541

Tazi, I., Nafil, H., and Mahmal, L. (2011). Monoclonal antibodies in hematological malignancies: past, present and future. J. Cancer Res. Ther. 7, 399-407. doi:10.4103/0973-1482.91999

Tholouli,E.,Hoyland,J. A.,DiVizio,D.,O'Connell,F.,MacDermott,S.A., Twomey,D., et al. (2006). Imaging of multiple mRNA targets using quantum dot based in situ hybridization and spectral deconvolution in clinical biopsies. Biochem. Biophys. Res. Commun. 348, 628-636. doi:10.1016/j.bbrc.2006.07.122

Tiacci, E., Park, J. H., De Carolis, L., Chung, S. S., Broccoli, A., Scott, S., et al. (2015). Targeting mutant BRAF in relapsed or refractory hairy-cell leukemia. N. Engl. J. Med. 373, 1733-1747. doi:10.1056/NEJMoa1506583

Tinkle, S., Mcneil, S. E., Mühlebach, S., Bawa, R., Borchard, G., Barenholz, Y. C., et al. (2014). Nanomedicines: addressing the scientific and regulatory gap. Ann. N. Y. Acad. Sci. 1313, 35-56. doi:10.1111/nyas. 12403

Venkatraman, S. (2014). Has nanomedicine lived up to its promise? Nanotechnology 25, 372501. doi:10.1088/0957-4484/25/37/372501

Vinhas, R., Cordeiro, M., Mendo, S., Carlos, F., Fernandes, A., Figueiredo, S., et al. (2015). Gold nanoparticle-based theranostics: disease diagnostics and treatment using a single nanomaterial. Nanobiosensors Dis. Diagnosis 4, 11-23. doi:10.2147/NDD.S60285

Vinhas, R., Cordeiro, M., Pedrosa, P., Fernandes, A. R., and Baptista, P. V. (2017a). Current trends in molecular diagnostics of chronic myeloid leukemia. Leuk. Lymphoma 58, 1791-1804. doi:10.1080/10428194.2016.1265116

Vinhas, R., Fernandes, A. R., and Baptista, P. V. (2017b). Gold nanoparticles for BCR-ABL1 gene silencing: improving tyrosine kinase inhibitor efficacy in chronic myeloid leukemia. Mol. Ther. Nucleic Acids 7, 408-416. doi:10.1016/j. omtn.2017.05.003

Vinhas, R., Correia, C., Ribeiro, P., Lourenço, A., Botelho de Sousa, A., Fernandes, A. R., et al. (2016). Colorimetric assessment of BCR-ABL1 transcripts in clinical samples via gold nanoprobes. Anal. Bioanal. Chem. 408, 5277-5284. doi:10.1007/ s00216-016-9622-9

Wetzler, M., Thomas, D. A., Wang, E. S., Shepard, R., Ford, L. A., Heffner, T. L., et al. (2013). Phase I/II trial of nanomolecular liposomal annamycin in adult patients with relapsed/refractory acute lymphoblastic leukemia. Clin. Lymphoma Myeloma Leuk. 13, 430-434. doi:10.1016/j.clml.2013.03.015
Winkler, I. G., Barbier, V., Nowlan, B., Jacobsen, R. N., Forristal, C. E., Patton, J. T., et al. (2012). Vascular niche E-selectin regulates hematopoietic stem cell dormancy, self renewal and chemoresistance. Nat. Med. 18, 1651-1657. doi:10.1038/nm.2969

Xu, X., Zhang, Y., Li, W., Miao, H., Zhang, H., Zhou, Y., et al. (2014). Wogonin reverses multi-drug resistance of human myelogenous leukemia K562/ A02 cells via downregulation of MRP1 expression by inhibiting Nrf2/ ARE signaling pathway. Biochem. Pharmacol. 92, 220-234. doi:10.1016/j. bcp.2014.09.008

Yan, Y., Fu, J., Wang, T., and Lu, X. (2017). Controlled release of silyl ether camptothecin from thiol-ene click chemistry-functionalized mesoporous silica nanoparticles. Acta Biomater. 51, 471-478. doi:10.1016/j.actbio.2017.01.062

Yang, H., Hui, H., Wang, Q., Li, H., Zhao, K., Zhou, Y., et al. (2014). Wogonin induces cell cycle arrest and erythroid differentiation in imatinib-resistant K562 cells and primary CML cells. Oncotarget 5, 8188-8201. doi:10.18632/ oncotarget. 2340

Yao, Q., Cao, F., Feng, C., Zhao, Y., and Wang, X. (2016). SERS detection and targeted ablation of lymphoma cells using functionalized Ag nanoparticles. Proc. SPIE 9724, Plasmonics in Biology and Medicine XIII, 972407. doi:10.1117/12.2212285

Young, R. M., and Staudt, L. M. (2013). Targeting pathological B cell receptor signalling in lymphoid malignancies. Nat. Rev. Drug Discov. 12, 229-243. doi: $10.1038 /$ nrd 3937

Yu, Y., Duan, S., He, J., Liang, W., Su, J., Zhu, J., et al. (2016). Highly sensitive detection of leukemia cells based on aptamer and quantum dots. Oncol. Rep. 36, 886-892. doi:10.3892/or.2016.4866

Zaimy, M., Saffarzadeh, N., Mohammadi, A., Pourghadamyari, H., Izadi, P., Sarli, A., et al. (2017). New methods in the diagnosis of cancer and gene therapy of cancer based on nanoparticles. Cancer Gene Ther. 24, 233-243. doi:10.1038/ cgt.2017.16

Zhan, J., Ma, Z., Wang, D., Li, X., Li, X., Le, L., et al. (2017). Magnetic and pH dual-responsive mesoporous silica nanocomposites for effective and low-toxic photodynamic therapy. Int. J. Nanomedicine 12, 2733-2748. doi:10.2147/IJN. S127528

Zhang, W., Xia, W., Lv, Z., Ni, C., Xin, Y., and Yang, L. (2017). Liquid biopsy for cancer: circulating tumor cells, circulating free DNA or exosomes? Cell. Physiol. Biochem. 41, 755-768. doi:10.1159/000458736

Zhou, S., Wu, D., Yin, X., Jin, X., Zhang, X., Zheng, S., et al. (2017). Intracellular $\mathrm{pH}$-responsive and rituximab-conjugated mesoporous silica nanoparticles for targeted drug delivery to lymphoma B cells. J. Exp. Clin. Cancer Res. 36, 24. doi:10.1186/s13046-017-0492-6

Zimmermann, M., Oehler, C., Mey, U., Ghadjar, P., and Zwahlen, D. R. (2016). Radiotherapy for non-Hodgkin's lymphoma: still standard practice and not an outdated treatment option. Radiat. Oncol. 11, 1-10. doi:10.1186/ s13014-016-0690-y

Zong, H., Sen, S., Zhang, G., Mu, C., Albayati, Z. F., Gorenstein, D. G., et al. (2016). In vivo targeting of leukemia stem cells by directing parthenolide-loaded nanoparticles to the bone marrow niche. Leukemia 30, 1582-1586. doi:10.1038/ leu.2015.343

Conflict of Interest Statement: The authors declare that the research was conducted in the absence of any commercial or financial relationships that could be construed as a potential conflict of interest.

The reviewer $\mathrm{CM}$ and the handling editor declared their shared affiliation.

Copyright $\odot 2017$ Vinhas, Mendes, Fernandes and Baptista. This is an open-access article distributed under the terms of the Creative Commons Attribution License (CC BY). The use, distribution or reproduction in other forums is permitted, provided the original author(s) or licensor are credited and that the original publication in this journal is cited, in accordance with accepted academic practice. No use, distribution or reproduction is permitted which does not comply with these terms. 